\title{
Breast panniculitis with liquefactive fat necrosis: A case report
}

\author{
YONGFENG ZHANG, XUHUA SHI and YUEWU LU \\ Department of Rheumatology and Immunology, Beijing Chao-Yang Hospital, \\ Capital Medical University, Beijing 100020, P.R. China
}

Received October 12, 2017; Accepted February 1, 2018

DOI: $10.3892 /$ etm.2018.6095

\begin{abstract}
Panniculitis is a group of heterogeneous disorders characterized by inflammation of the subcutaneous adipose tissue. Panniculitis of breast tissue as the initial manifestation has rarely been reported and is often misdiagnosed. Breast panniculitis may cause substantial morbidity and early diagnosis and treatment are important for the prognosis of the disease. The present study has reported a case of panniculitis with inflammation of the mammary glands as the initial presentation and provided a detailed description of ultrasonography, X-ray, computed tomography, magnetic resonance imaging and other imaging features of breast panniculitis. The treatment and follow-up were also described. Following treatment with systemic corticosteroids combined with methotrexate and thalidomide for 2 months, the breast appeared to be normal without scar formation. The present case report provides a good reference for the future diagnosis and treatment of breast panniculitis.
\end{abstract}

\section{Introduction}

Panniculitis is a group of heterogeneous inflammatory disorders of the subcutaneous adipose tissue. The diagnosis of panniculitis requires histopathologic examination. Clinically, patients with panniculitis frequently present with multiple palpable nodules on the trunk or lower extremities. According to the predominant location of the microscopic inflammation determined by histopathology, panniculitis can be divided into two subtypes: Septal or lobular $(1,2)$. However, panniculitis of breast tissue as the first manifestation has rarely been reported and is often misdiagnosed as mastitis, or even breast cancer during clinical examination. Without proper and timely treatment, breast panniculitis can cause significant morbidity. First, at acute inflammatory phase, breast becomes painful. Later the skin develops ulceration and infection. At chronic phase, the inflammation is resolved, but breast can be left with deep atrophic

Correspondence to: Dr Yuewu Lu, Department of Rheumatology and Immunology, Beijing Chao-Yang Hospital, Capital Medical University, 8 Gongren Tiyuchang Nanlu, Beijing 100020, P.R. China E-mail: sxhherosci@sina.com

Key words: panniculitis, breast, erythema nodosum scars, cosmetic disfigurement, which may lead to psychiatric sequelae for some patients (3). So, early diagnosis and treatment are very important for the prognosis of breast panniculitis. Hereby we reported a patient suffered from the liquefactive fat necrosis of breast tissue, a rare case of panniculitis who first presented with irregular breast nodules, followed by erythema nodosum of both lower extremities, to help understand the early presentation of breast panculitis for future diagnosis.

\section{Case report}

A 37-year-old woman without a relevant medical history presented with fever and recently occurred painful subcutaneous nodules predominantly in the upper quadrants of his left breast. Physical exam identified an area of poorly-defined, marked induration and tenderness on her breast, with inflammatory changes on the skin but without nipple discharge or retraction (Fig. 1). The right breast appeared normal. The highest body temperature was $38.5^{\circ} \mathrm{C}$.

The patient was initially seen in the Department of Surgeon. At the first visit, physical examination showed tenderness and irregular subcutaneous nodules at the upper inner quadrant of her left breast, accompanied by mild local skin inflammation, without nipple discharge and swelling of axillary lymph nodes. The patient lacks any history of breast trauma, tumor and connective tissue diseases. X-ray of her left breast showed no clear abnormalities of the skin, nipple and subcutaneous adipose tissue. Several patchy hyperintense signals, linear streaky shadows and fuzzy nodules were seen at the left breast. All signals were evenly dispersed and scattered calcifications were also visible. There was no localized soft tissue mass and clustered micro-calcification in the left breast tissue (Fig. 2). The patient was first treated with cefaclor (Sustained Release Tablets, $0.375 \mathrm{~g}$ orally twice daily) and topical drugs lactate ethacridine solution, twice daily for 3 months. However, the treatment was not effective. Subsequently, she underwent breast ultrasonography. The pattern of left breast tissue was clear, the echo of the glands was uneven, mixed with strong and weak signals, and there was no expansion of the breast duct. A reduced echo of subcutaneous soft tissue was shown at the upper inner quadrant of her left breast. The area was approximately $4.4 \times 1.5 \mathrm{~cm}$ in size, without a clear margin, and the echo was not uniform, indicating abundant blood flow but a lack of clear boundary with the rear glands. The rear glands also showed a reduced and uneven echo without evident mass, and significant local tenderness (Fig. 2B). 
After three months of antibacterial and topical treatment, dark reddish plaques and tender skin lesions were identified over both legs, 3-4 cm in size (Fig. 3). The patient was then referred to the Department of Rheumatology and Immunology. At the time of visit, the patient was depressed and admitted for further evaluation. Upon admission, initial laboratory results revealed normal blood count, increased erythrocyte sedimentation rate $(27 \mathrm{~mm} / \mathrm{h})$ and C-reactive protein $(1.05 \mathrm{mg} / \mathrm{ml})$. Pro-calcitonin, $\beta$-D-glucan, PPD-test, T-SPOT were all negative. Breast puncture fluid was purulent (Fig. 1C). The puncture fluid routine test showed white blood cell of 40/HPF, and negative on bacteria fungus mycobacterium tuberculosis infection from the puncture fluid culture. Histopathological findings of lower limb skin and subcutaneous tissue showed no obvious atypicality of epithelial cells, mild proliferation of subepithelial fibrous tissue, the distribution of granuloma composed of epithelioid cells and multinucleated giant cells along the leaflet interval. Immunohistochemical staining: acid-fast staining, PAS and PASM were all negative.

The pathological results indicated septal panniculitis, known as erythema nodosum (Fig. 3C). ANA titer was 1:320, but the results of anti-dsDNA, anti-CCP antibody, antineutrophil cytoplasmic antibody (ANCA), anti-phospholipid antibody, immunoglobulin G, complement factors $\mathrm{C} 3$ and $\mathrm{C} 4$ were all within the normal range. Chest computed tomography (CT; Fig. 2C) showed thickening of the left breast skin, rugged local skin, increased density of subcutaneous adipose tissue, CT value of 10 . The density of left breast tissue was not uniform, and the normal gland structure also disappeared. Left breast magnetic resonance imaging (MRI) also showed the disappearance of normal gland structure. T2-weighted image (T2WI) imaging revealed small focal lesions, large areas of irregular abscess-like changes, most significant within the inner upper quadrant, outer lower quadrant and behind the areola. The lesions at the inner upper quadrant also involved the subcutaneous fat and skin, showing local skin thickening with rugged surface and abscess formation connected with the glands, as well as the retraction of left nipple (Fig. 2D). DWI imaging showed dotted, patchy and annular high signals (Fig. 2E). Dynamic enhancement imaging revealed the flake-like and circular lesions, with large irregular abscess walls that were shown as mild to moderate early enhancement, and strengthened late continuous enhancement (Fig. 2F).

According to the clinical presentation, laboratory, imaging and pathological results, the patient was diagnosed as breast panniculitis with liquefactive fat necrosis. The patient received systemic corticosteroids, intravenous methylprednisolone $80 \mathrm{mg}$ daily for 3 days, following oral prednisone $60 \mathrm{mg}$ daily, combined with methotrexate $(12.5 \mathrm{mg} / \mathrm{W})$ and thalidomide $(50 \mathrm{mg} /$ day).

After 3 days of treatment, the patient's body temperature returned to normal, nodular erythema of lower extremities, and breast tenderness all significantly subsided. After 1 month of treatment, glucocorticoids were tapered off. After 2 months of treatment, mastoid nodules and lower extremity nodules erythema disappeared (Figs. 1B and 3B). The breast appeared to be normal without scar formation. The patient's anxiety and depression were also improved. The patient provided written informed consent for the publication of their data.

\section{Discussion}

Panculitis is a rare inflammatory disorder of subcutaneous adipose tissue. The characteristic painful nodules are observed primarily in the area of the lower extremities and the trunk (4). However, the breast can also be seldom involved. For mammary glanditis the imaging study and systematic treatment are rarely reported.

Breast panniculitis is characterized by subcutaneous painful mass of the breast, accompanied by fever and fatigue and other systemic inflammation, as well as depression especially in women. This paper reported a case of papillitis with mammary gland involvement as the early presentation, and provided a detailed description of ultrasonography, X-ray, CT, MRI and other imaging findings of breast panniculitis. The treatment of breast panniculitis and follow-up were also recorded in detail. Together this case report provided a good reference for the future diagnosis and treatment of breast panniculitis. In clinical practice when the patient presents with breast pain, in addition to the commonly seen suppurative mastitis and tumor, papillitis should also be taken into consideration.

Breast nodule within a few centimeters in size may be visualized by ultrasound. The imaging findings of ultrasonography have been shown to be associated with the stage of disease and the degree of fibrosis $(5,6)$. In the acute inflammatory stage, adipocytes are destroyed or necrotized, accompanied by the infiltration of inflammatory cells. The ultrasound imaging features high echo nodules with internal hypoechoic cyst or no echo capsule, and unevenly echoed tissue shadow. In the recovery and chronic phase, along with tissue fibrosis, ultrasound imaging shows diffused high echo mass, and lesions with margins that can be clear, fuzzy or needle-shaped depending on the degree of fibrosis (7).

Breast malignancy typically features: i) Irregular shaped tumor with spicule sign and lobular sign; clustered or sandy like micro-calcifications; invasion of local skin, subcutaneous fat layer and chest wall, and axillary lymph node enlargement. ii) Blood flow increase in and around the nodule (8). The breast lesion of our patient did not present these features. Rather, the sonography report suggested inflammatory lesions. This result suggested the breast lesion was inflammation.

It has also been reported that for the diagnosis of breast panniculitis, mammography is superior to ultrasonography, especially for evaluating early micro calcification. Early calcifications frequently resemble malignancy and might present in a ductal distribution. In our case, breast X-ray imaging showed local mass shadow that could be penetrated by the X-ray, there was no apparent calcification, and fat necrosis is also shown as the penetrable cavity. The variable mammography features can be attributed to the degree of fibrosis in different stages of panculitis $(6,9)$. Characteristic presentation of breast malignancy include: local invasion, spicule sign, clustered calcifications; local skin thickened, nipple retraction, etc. The mammography of our patient showed patchy hyper densities, to which the radiologist suggested further evaluation.

We noted the breast lesion on the CT. The lesion was an irregular shaped mass. However, non-contrast chest CT is 

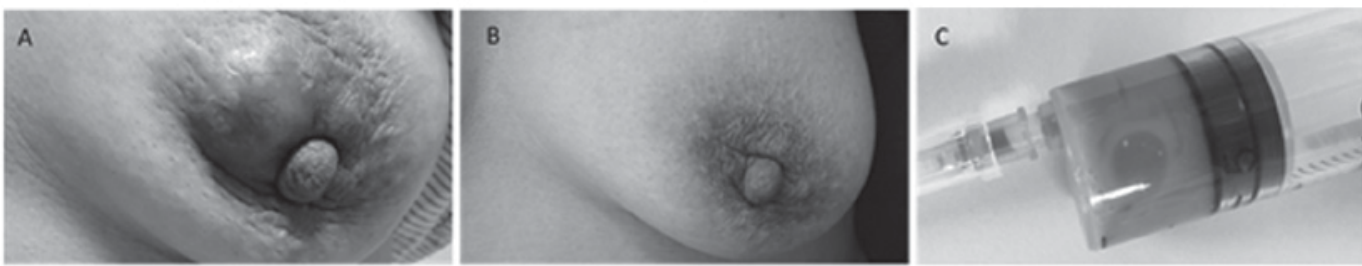

Figure 1. (A) Irregular subcutaneous nodules at the inner upper quadrant of the left breast, accompanied by local skin inflammation. (B) After 2 months treatment, nodules and local skin inflammation subsided. (C) Purulent fluid from the left breast puncture.
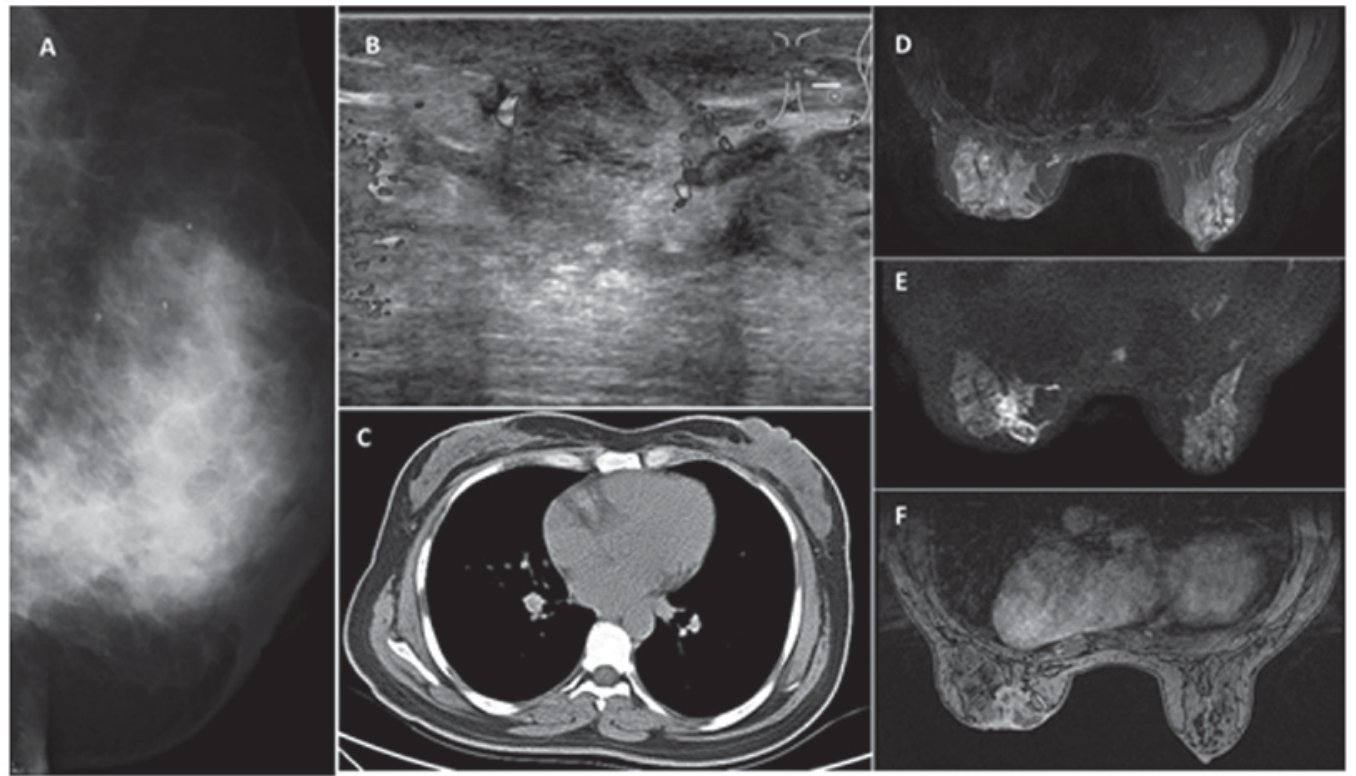

Figure 2. (A) Mammography, multiple patchy hyperintense signals, linear shadows and fuzzy nodules at the left breast. All signals were evenly dispersed. (B) Breast ultrasound, gland echo is not homogeneous, mixed with strong and weak signals, no expansion of the breast duct, a reduced echo of subcutaneous soft tissue at the upper inner quadrant. The area was approximately $4.4 \times 1.5 \mathrm{~cm}$ in size, without a clear margin, and the echo was not uniform. (C) Breast computed tomography, left breast skin thickening, uneven density of breast tissues and destruction of normal gland structure. (D) Breast MRI, T2WI imaging, focal, irregular sized abscess shadow. (E) Breast MRI diffusion-weighted sequence, focal, patchy and circular signal. (F) Breast MRI dynamic enhancement imaging, flake-like and circular lesion signals, and large irregular abscess walls. MRI, magnetic resonance imaging; T2WI, T2-weighted image.

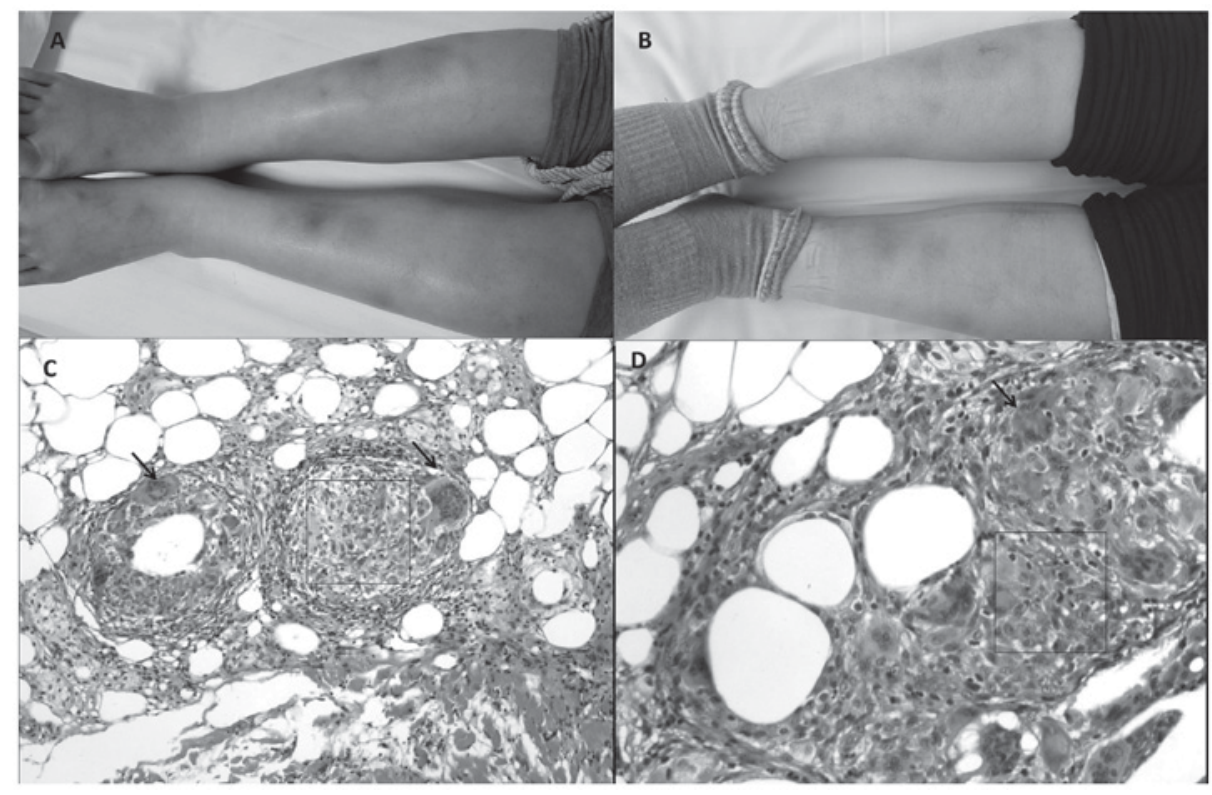

Figure 3. (A) Nodular erythema of both lower extremities. (B) After 2 months treatment, the nodular erythema disappeared, left with residual pigmentation. (C and D) Skin histopathology: lack of atypical epithelial cells, hyperplasia of subepithelial fibrous tissue, the granuloma tissue (square area) constitutes of epithelioid cells and multinucleated giant cells (arrow area) distributed along the leaflets interval (hematoxylin and eosin staining, (C) magnification, x200; (D) magnification, $\mathrm{x} 400$ ). 
insufficient to differentiate between benign and malignant breast lesions (10). Contrast-enhanced breast CT might clearly display the lesion size, location, density, appearance, blood vessel around the lesion, and provide better differential diagnosis for early breast cancer. Unfortunately, our patient only underwent high-resolution CT scans.

Magnetic resonance imaging has used high spatial resolution techniques for soft tissue evaluations, and has unique contributions for the diagnosis of breast disease as a radiation-free and no-invasive method. To our knowledge, the MRI of breast panniculitis has rarely been reported in the literature. Pinho et al found that on MRI imaging, breast panculitis was shown as a large irregular-shaped, heterogeneous shadow with high signal intensity on T2-weighted images, medium signal intensity on T1-weighted images, and regional enhancement. These features are nonspecific and can be attributed to inflammatory process and edema (9). Kinetic analysis also showed continuous enhancement, suggesting a benign lesion. For the early stage of panculitis accompanied by focal edema, MRI shows hyperintense signal on T2-weighted images. The stage of fat necrosis consists of oil cysts, which can be shown as circular, well-circumcised hyperintense areas on T1-weighted images (11). Our patient presented nodular, patchy, small annular and large irregular cavities and hyperintense signal on T2-weighted images, and circular, focal, patchy hyperintense signal on DWI. These features suggested inflammatory and benign nature of the lesions. But at the final stage of the disease, panculitis could present with indistinct or speculated margins and structural distortion on MRI, which may be indistinguishable from breast malignancy.

Histopathology can also be helpful to ascertain the type of breast panniculitis, distinguish it from other lesions, and guide the further therapy. The diagnosis of breast panniculitis is different. However, upon careful review of histopathology, a specific diagnosis can be appropriately made. First, we should know that breast panniculitides are mixed panniculitis because of inflammatory infiltration involving both the septa and the lobules of the subcutaneous adipose tissue. Second, the pathological examination is to assess whether vasculitis is present. Third, it is necessary to identify the nature of the inflammatory infiltration and additional histopathologic features of the lesions, in order to draw a specific diagnosis of the disease that involves the subcutaneous fat $(12,13)$. Our patient mostly presented fat necrosis of left breast. According to the characteristics of adipocytes necrosis, there are five histopathologic types, including lipophagic necrosis, liquefactive fat necrosis, hyalinizing fat necrosis, membranous fat necrosis, and ischemic fat necrosis (14). With regard to our patient, the extracted fatty tissue was liquefactive, but etiological examination was negative. Therefore, the lesion was considered mostly lobular panniculitis with liquefactive fat necrosis. The presence of vasculitis and nature of infiltrated cells were not assessed.

At present, there is no established therapeutic strategies for breast panniculitis. Many patients underwent surgical treatment. Therefore, the early diagnosis and timely treatment is very important, to avoid surgery and irreversible damage of the breast. Firstly, lobular panniculitis is generally resulted from systematic diseases, such as systemic lupus erythematosus, pancreatic diseases, $\alpha 1$-antitrypsin deficiency, infections, trauma, etc. So, the treatment of root causes is critical. When the etiology cannot be determined, such as in this case, panniculitis is termed as idiopathic. According to pathological characteristics, a large number of inflammatory cells were present in the lesion. Idiopathic panniculitis refers to a condition of non-infective fat tissue inflammation. Rotaru et al reported a case of non-suppurative nodular breast panniculitis with systemic symptoms. After treated with systemic corticosteroids, the condition improved (15). Yuan reported a case of isolated breast panniculitis without evidence of systemic inflammation, who underwent resection without systemic corticosteroid or immunosuppressive therapy (16). The patient did not suffer a relapse. Others also reported the isolated breast panniculitis cases that had an excellent prognosis with resection only $(17,18)$. Our patient was complicated by fever, nodular erythema of both lower extremities, and ESR elevation. Although the lesion was punctured and drained, the patient's overall symptoms had not improved. Therefore, we gave the patient experimental glucocorticoid therapy, and follow-up with immunosuppressive therapy. Her general condition, breast lesions, lower extremity erythema all markedly improved as a result. So far, the patient has not had a relapse.

Breast panniculitis is a rare condition, can be mistaken for cancer at clinical examination, frequently associated with systemic symptoms and occasional visceral involvement. Through histopathology and imaging examinations, it can be diagnosed and treated early, to avoid further visceral involvement and breast disfiguration. From the experience of our patient, glucocorticoids combined with immunosuppressive therapy could prevent the relapse.

\section{Competing interests}

The authors declare that they have no competing interests.

\section{References}

1. Requena L and Sanchez Yus E: Panniculitis. Part II. Mostly lobular panniculitis. J Am Acad Dermatol 45: 325-364, 2001.

2. Requena L and Yus ES: Panniculitis. Part I. Mostly septal panniculitis. J Am Acad Dermatol 45: 163-186, 2001.

3. Grossberg E, Scherschun L and Fivenson DP: Lupus profundus: Not a benign disease. Lupus 10: 514-516, 2001.

4. Chowaniec M, Starba A and Wiland P: Erythema nodosum-review of the literature. Reumatologia 54: 79-82, 2016.

5. Taboada JL, Stephens TW, Krishnamurthy S, Brandt KR and Whitman GJ: The many faces of fat necrosis in the breast. AJR Am J Roentgenol 192: 815-825, 2009.

6. Tan PH, Lai LM, Carrington EV, Opaluwa AS, Ravikumar KH, Chetty N, Kaplan V, Kelley CJ and Babu ED: Fat necrosis of the breast-a review. Breast 15: 313-318, 2006.

7. Ng AM, Dissanayake D, Metcalf C and Wylie E: Clinical and imaging features of male breast disease, with pathological correlation: A pictorial essay. J Med Imaging Radiat Oncol 58: 189-198, 2014.

8. Tan TJ, Leong LC and Sim LS: Clinics in diagnostic imaging (147). Male breast carcinoma. Singapore Med J 54: 347-352, 2013.

9. Pinho MC, Souza F, Endo E, Chala LF, Carvalho FM and de Barros N: Nonnecrotizing systemic granulomatous panniculitis involving the breast: Imaging correlation of a breast cancer mimicker. AJR Am J Roentgenol 188: 1573-1576, 2007.

10. Bach AG, Abbas J, Jasaabuu C, Schramm D, Wienke A and Surov A: Comparison between incidental malignant and benign breast lesions detected by computed tomography: A systematic review. J Med Imaging Radiation Oncol 57: 529-533, 2013. 
11. Kuhl CK, Mielcareck P, Klaschik S, Leutner C, Wardelmann E, Gieseke J and Schild HH: Dynamic breast MR imaging: Are signal intensity time course data useful for differential diagnosis of enhancing lesions? Radiology 211: 101-110, 1999.

12. Ferrara G, Stefanato CM, Gianotti R, Kubba A and Annessi G: Panniculitis with vasculitis. G Ital Dermatol Venereol 148: 387-394, 2013.

13. Wick MR: Panniculitis: A summary. Semin Diagn Pathol 34: 261-272, 2017.

14. White WL, Wieselthier JS and Hitchcock MG: Panniculitis: Recent developments and observations. Semin Cutan Med Surg 15: 278-299, 1996.

15. Rotaru N, Punga J, Codreanu I, Gavrilasenco I, Manea D and Cujba N: Nonsuppurative nodular panniculitis of the breast. Clin Breast Cancer 15: e219-e221, 2015.
16. Yuan WH, Li AF, Hsu HC and Chou YH: Isolated panniculitis with vasculitis of the male breast suspicious for malignancy on CT and ultrasound: A case report and literature review. Springerplus 3: 642, 2014.

17. Hernandez-Rodriguez J, Tan CD, Molloy ES, Khasnis A, Rodriguez ER and Hoffman GS: Vasculitis involving the breast: A clinical and histopathologic analysis of 34 patients. Medicine (Baitimore) 87: 61-69, 2008.

18. Kafantari E, Sotiropoulou M, Sfikakis P, Dimitrakakis K, Zagouri F, Mandrekas K, Dimopoulos S, Dimopoulos MA and Papadimitriou CA: Giant cell arteritis of the breast and breast cancer: Paraneoplastic manifestation or concomitant disease? A case report. Onkologie 31: 685-688, 2008. 